\title{
Direct cholangioscopy with argon plasma coagulation of an intraductal papillary mucinous neoplasm of the bile duct
}

\author{
Boram Cha ${ }^{1}$, Jin-Seok Park ${ }^{1}$, Seok Jeong ${ }^{1}$, Don Haeng Lee ${ }^{1}$, and Joon Mee Kim²
}

${ }^{1}$ Digestive Disease Center, Department of Internal Medicine, ${ }^{2}$ Department of Pathology, Inha University School of Medicine, Incheon, Korea
Received: August 30, 2017

Revised : September 11, 2017

Accepted: November 11, 2017

\section{Correspondence to}

Jin-Seok Park, M.D.

Tel: +82-32-890-2548

Fax: +82-32-890-2549

E-mail: pjs@inha.ac.kr
An 81-year-old man was admitted for abdominal pain, fever, and jaundice. Contrast enhanced computed tomography (CT) showed marked dilation of both intra- and extrahepatic ducts (Fig. 1A). Endoscopic retrograde cholangiopancreatogram suggested a diagnosis of intraductal papillary mucinous neoplasm from the bile duct (IPMN-B) due to abundant mucin that exuded from papilla and common bile duct dilatation with an amorphous filling defect (Fig. 1B). Direct per-oral cholangioscopy using a conventional upper endoscope (GIFQ260J, Olympus, Tokyo, Japan) was planned. Cholangioscopy revealed multiple exophytic papillary protrusions consistent with IPMN-B involving the left intrahepatic ducts and hilar portion, but an un- involved right intrahepatic duct (Fig. 2A). Cholangioscopy-directed biopsy was performed and papillary epithelial neoplasm with intermediate grade of dysplasia was diagnosed histologically. Argon plasma coagulation (APC) was performed at papillary lesions to reduce tumor burden with the goal of reducing mucin production. The lesions were successfully ablated by three sessions of APC performed over 8 days at $40 \mathrm{~W}$, pulsed, effect 1, and 1.0/min flow (Fig. $2 \mathrm{~B}$ and $2 \mathrm{C}$ ). One month later, CT showed marked improvements of intra-andextrahepatic duct dilations, and at 8 months after treatment the patient was still healthy without abdominal pain or recurrent cholangitis.

This case raises two aspects of clinical interest. First, no previous case
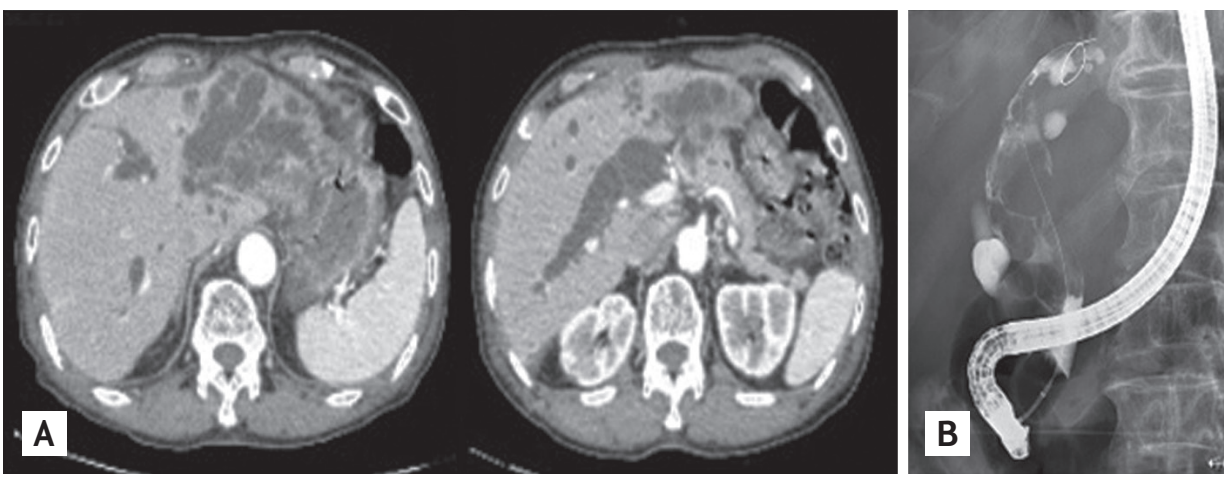

Figure 1. (A) Computed tomography showing diffuse extrahepatic and intrahepatic bile duct dilation. (B) Endoscopic retrograde cholangiogram showing severe dilatation of the common bile duct with amorphous filling defects. 

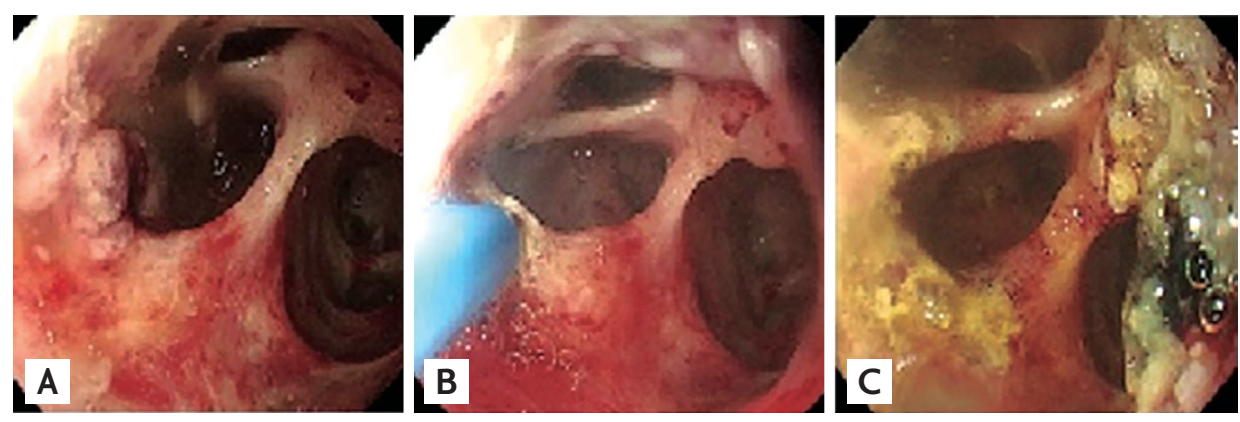

Figure 2. Endoscopic view of the bile duct. (A) Multiple protruding and friable mucosal lesions were observed at the left intrahepatic duct. (B) Argon plasma coagulation (APC) of mucosal lesions in left intrahepatic duct. (C) Appearance after APC.

report has been issued of a successfully diagnosed IPMN-B patient treated by direct per-oral cholangioscopy using a conventional upper endoscope. Second, no clear guideline has been issued regarding the palliative treatment of IPMN-B, and thus, we hope our experience may aid considerations of palliative treatment for similar patients.

In patients with IPMN-B, recurrent cholangitis is the most problematic situation, despite endoscopic biliary drainage. In our patient, APC reduced mucin production and effectively ablated almost all tumor tissues; the ablation of all tumor tissues was impossible because scope movement was limited in the bile duct.

Written informed consents were obtained.

\section{Conflict of interest}

No potential conflict of interest relevant to this article was reported.

\section{Acknowledgments}

The study was supported by an Inha University Research Grant. 